\title{
Semantic-driven 3D Scene Construction Based on Spatial Relationship and Case-base
}

\author{
1st Hui Liang \\ Department of Software \\ Engineering \\ Zhengzhou University of Light \\ Industry \\ Zhengzhou, China \\ hliang@zzuli.edu.cn
}

\author{
2nd Kailu Lv \\ Department of Software \\ Engineering \\ Zhengzhou University of Light \\ Industry \\ Zhengzhou, China \\ 1272741817@qq.com
Zhengzhou University of Light Industry
Zhengzhou, China
pmg-1@163.com
6th Jian Chang

5th Mingge Pan

School of Arts and Design

\author{
3rd Yusheng Sun \\ Department of Software \\ Engineering \\ Zhengzhou University of Light \\ Industry \\ Zhengzhou, China \\ 1987004@zzuli.edu.cn
}

\author{
Animation \\ Bournemouth University \\ Poole, UK \\ jchangsuper@163.com
}

\author{
4th Qian Zhang \\ School of Arts and Design \\ Zhengzhou University of Light \\ Industry \\ Zhengzhou, China \\ qianzhang@zzuli.edu.cn
}

\begin{abstract}
At present, 3D scene construction technology has been widely used in various fields. However, 3D scene construction is increasing on the human and material costs, and the production process is also complicated. First, we use a semantic analysis method to achieve better Chinese automatic word segmentation, which is bidirectional matching Chinese word segmentation based on $\mathrm{N}$-gram model. Next, to solve the problems of low degree of automation and intelligence, we propose a new method of $3 D$ scene construction based on spatial relationship and case-base. The objects and spatial relationships extracted from the scene description texts form a spatial constraint in the form of a triple, which is stored in the spatial relationship template library. Then the $3 \mathrm{D}$ model in the case-base is invoked to build the scene. This method takes the spatial constraint as the smallest module of the scene construction, which not only accelerates 3D scene construction, but also improves the rationality of the scene layout. At last, we apply this method to scene generation in a strategy game, in which the effectiveness and efficiency of the new method are proved.
\end{abstract} gram

Keywords-3D scenes, spatial relationships, case-base, $N$ -

\section{INTRODUCTION}

Three-dimensional scene construction technology uses digital image processing, computer graphics, computer vision, human-computer interaction, and other technologies to create a realistic virtual world through the powerful computing capabilities and graphics rendering ability of computers [1-6]. At present, 3D scene construction technology has been widely applied to military, medical, education, entertainment, urban planning, architectural design, cultural relics protection, and other fields. However, this work is clumsy and timeconsuming, which requires a large number of excellent $\mathrm{CG}$ (Computer Graphics) personnel. To reduce the labor and time costs of modeling, the automation of 3D scene construction technology is an inevitable trend. Many scholars make deeply research on this project.
In the production of the game and movie animation making, the scene design provides characters' activity environment in the play. The quality of scene production technology is directly related to the quality of film modeling. The basic content of scene design includes the construction of the basic scene and the selection and layout of props in the scene. With the rapid development of computer technology, people can complete a large number of heavy manual drawing work with the help of the technique of computer. Compared with the traditional method, in addition to the repeated modification in the design process, the design results can be reused and shared, which greatly improves the design efficiency and reduces the production costs. From the earliest spatial automatic layout based on geometric constraints to the scene construction based on semantics, from manual intervention to automatic generation of system, the research on automatic generation of scene has achieved many beneficial results. However, accurate scene object relationship description, efficient automatic and intelligent modeling method, and model sharing and reuse are still urgent difficulties in current 3D animation scene modeling field [7-8]. This paper proposes a new method of semanticdriven 3D scene construction based on spatial relation and case-base, and we apply this method to a strategy game.

Semantic analysis is the first step of computer processing after the user inputs data. Natural language processing (or Computational Linguistics) is becoming the most advanced technology in the world. It has been developed since the 1960s. Text mining, also known as "text analysis", uses natural language processing to transform unstructured corpus into standard and standardized documents or databases for further analysis through the application of artificial intelligence technology and machine learning algorithm [9]. Text mining includes a series of techniques for representing and transforming text. In-text mining, NLP is a collection of processing algorithms based on syntactic and semantic rules or statistics, which can be used to parse, segment, extract or analyze text data [10]. 
According to research, special games can increase players' brain flexibility, especially strategy games. Scientists call brain flexibility the foundation of human intelligence. Strategy games can be used to enhance the ability to solve problems, even beyond other abilities [11]. For education, Nikitakos [12] uses a-variety of strategies in the execution games. To better execute such games, this paper introduces semantic-driven 3D scene construction based on spatial relation and case-base. In the scene layout, the decision-making ability of the brain is trained to enhance its flexibility.

This paper is structured as follow. Section1 presents the research background, the research significance and content structure. Section2 presents some related works in the generation of a 3D scene and its application in strategy games. Section3 introduces Chinese word segmentation and spatial relation extraction. Section4 focuses on 3D scene construction method based on spatial relation and case-base. Section5 mainly discusses application in strategy game. Finally, Section6 presents our main conclusions and some ideas for future work.

\section{RELATED WORK}

3D scene design and synthesis has been widely used in game development and animation design. Fisher [13] proposes the user input 3D scene to generate some new 3D scene. Gallup [14] proposes an automatic scene reconstruction method based on video, which uses the algorithm of multi view depth map fusion to extract the height map of 3D surface. This paper proposes the semantic-driven 3D scene construction based on spatial relation and case-base in strategy games to improve the traditional 3D scene construction.

The rapid development of Computational Linguistics provides a new solution for 3D scene construction [15-17]. People can annotate parts of speech, syntactic analysis, and semantic frame of natural language, and develop information retrieval, information extraction, and other technologies [1822]. For natural language, the smallest unit of meaning is a word, while for Chinese, the basic unit is a character. Chinese natural language processing should include the processing of characters, words, sentences, paragraphs, and chapters [23]. Due to the Chinese language particularity, Chinese word segmentation has developed into an independent research field, and many knowledge theories and algorithms have emerged. The corresponding research results of Chinese word segmentation are applied to different tasks by natural language processing, including information retrieval, machine translation, speech recognition, text error recognition, traditional and simplified Chinese automatic conversion, automatic answering question, and so on [24-28]. At present, in the process of actual word segmentation, there are three key problems: the standardization of word segmentation, the segmentation of ambiguous words, and the recognition of unknown words [2930]. According to different research methods, the existing word segmentation algorithms can be divided into three categories: dictionary-based word segmentation, statistical-based word segmentation, and rule-based word segmentation. The main models of word segmentation based on statistics are $\mathrm{N}$-gram model and Hidden Markov model (HMM). In this paper, the semantic analysis is based on the $\mathrm{N}$-gram model.
At present, the methods of scene construction can be divided into the following directions: constraint-based scene construction, rule-based scene construction, and learning-based scene construction.

Scene construction based on constraints. The earlier ones are the MultiCAD-GA system designed by Vassilas [31] and the DEM2OMS system designed by Ghassan [32]. Both systems set constraints in advance, and use a genetic algorithm to solve the furniture layout problem. The difference is the way to deal with the initial layout solution. The former uses constraint programming technology; the latter uses domain filtering technology to reduce the number of initial placement. In 2002, Xu Ken and others of Toronto University developed the CAPS system. The system uses geometric constraints based on the virtual physical world and semantic constraints based on the position relationship between objects in the real world to automatically guide the placement of objects in spatial $[33,34]$. In 2014, Liu Yongshan publishes a method for selecting and invoking LOD models of 3D scenes based on double constraints, which combines the distance constraints to select and invoke the level of objects detail models needed by users. After Bukowski proposes object association constraint, many scholars developed different furniture layout systems based on this concept [35-37]. This kind of system mainly aims at the relationship between objects and establishes some constraints that the object layout needs to satisfy, such as physical constraints, geometric constraints, surface contact constraints, and so on. However, it lacks consideration on the function, applications and overall layout of objects.

Scene construction based on rules. Chang [38] analyzes the relationship between objects in real life. For example, there are chairs beside the desk and books on the desk. According to these relations, the corresponding scene hierarchy diagram is established. According to this, establish the corresponding rules, according to these rules to guide the furniture placement. In 2002, academician Lu Ruqian establishes a series of rules manually, such as trees, flowers, animals, and so on, and enriches the default scenes according to these rules.

Scene construction based on learning. Stanford University [39] is a representative as learning based scene construction. It has developed an indoor 3D scene object placement system that can infer implicit relationships. The system can support interactive scene manipulation and active learning. In 2017, Liu Milan proposes an indoor furniture layout method based on a case-base [40]. Firstly, the interior contour structure feature is defined. Then, the regions are taken as the layout object, and the prior knowledge is extracted from the case-base. Finally, the relationship between the regions and the interior contour structure features is established. Funkhouser proposes a method of 3D modeling based on examples. Users can retrieve the 3D model database to get different parts of the model, and use different combinations of different parts of the model to build a new 3D geometric model.

However, the application of $3 \mathrm{D}$ scene construction is relatively less in strategy game. The strategy game has always been recognized as a game that helps to train thinking. In the game, players need careful thought to deal with unexpected turning points at any time, relying on the use of strategy to 
defeat opponents. As we all know, the Three Kingdoms have a magnificent historical background, which also produced many influential characters and classic stories. The main stories, events, and role descriptions in the novel are useful and transferable materials for computer games, which makes the romance of the Three Kingdoms incarnate as a high-tech product and widely spread in the game. For example, Yingshu Cai [41] suggests that the description based on the main characters and events in the novel, players can construct the design of scene type, character, and background. For example, Shiang-Kwei [42] studies whether games and learning outcomes are positively correlated, and puts forward the factors that help to improve the development of players' literary quality as well as how players design scenes according to the story of the romance of the Three Kingdoms. The results show those game players have a better understanding of the history of the Three Kingdoms period to increase their interest in history learning. Therefore, the directed game can play a positive role for the players. It is possible to take important stories of the Three Kingdoms period as the background of the game and to exercise people's thought from strategy games.

In this paper, a 3D scene construction method based on a spatial relation case-base is proposed. This method improves the usability and quickness of 3D scene production and makes players pay more attention to how to use strategies to arrange the scene in the game to beat the opponent.

\section{CHINESE WORD SEGMENTATION AND SPATIAL RELATIONSHIP EXTRACTION}

The elements of information contents mainly include individual, relationship, feature, situation, and spatial location. The elements in the sentence extracted from scene semantic information include subject, subject attribute, predicate, topic, tool, time, and so on. The following contents introduce how to extract semantic information through lexical analysis and spatial semantic analysis.

\section{A. Bidirectional matching Chinese word segmentation method based on N-gram model}

In the method of word segmentation based on statistical learning, the $\mathrm{N}$-gram statistical language model uses the relevant information between adjacent words in context to select the word combination with the maximum occurrence probability to realize automatic word segmentation [43]. The model is language independent, strong spelling compatibility, and can deal with Chinese, English, traditional, and simplified texts. It is a common statistical language model for Chinese information processing. By combining the $\mathrm{N}$-gram model with the dictionary and combining the advantages of statistical learning and dictionary matching, Chinese automatic word segmentation can be performed better. Based on the $\mathrm{N}$-gram language model, this paper uses a Bidirectional matching Chinese word segmentation method.

The Chinese word segmentation method based on the Ngram model extends the Markov hypothesis of the $\mathrm{N}$-gram model. Note $\mathrm{S}=c_{1} c_{2} \ldots c_{N}$ is the Chinese character string to be segmented, and $c_{j}$ is the Chinese character, $1 \leq \mathrm{j} \leq \mathrm{N}$. $l=\left(w_{1}^{\prime}, w_{2}^{\prime}, \ldots, w_{M}^{\prime}\right)$ is a possible segmentation result of $\mathrm{S}$, $1 \leq \mathrm{M} \leq \mathrm{N}$. If the appearance of the kth word $w_{k}^{\prime}$ only depends on the $\mathrm{n}-1$ words before it, then According the hypothesis of the n-1 order Markov, the probability formula of $w_{k}^{\prime}$ is as follows:

$$
P\left(w_{k}^{\prime} \mid w_{1}^{\prime}, w_{2}^{\prime}, \ldots, w_{k-1}^{\prime}\right)=P\left(w_{k}^{I} \mid w_{k_{\mathrm{n}}+1}^{\prime}, w_{k_{\mathrm{n}}+2}^{\prime}, \ldots, w_{k-1}^{\prime}\right)(1)
$$

$k_{0}=\max (\mathrm{k}-\mathrm{n}, 0)$. The probability of sentence $w_{1}^{\prime}, w_{2}^{\prime}, \ldots, w_{M}^{\prime}$ composed of sequence $l=\left(w_{1}^{\prime}, w_{2}^{\prime}, \ldots, w_{M}^{\prime}\right)$ with $\mathrm{M}$ words is as follows:

$$
P(l)=\prod\left(w_{1}^{I}\right) \prod_{k=2}^{M} P\left(w_{k}^{\prime} \mid w_{k_{0}+1}^{\prime}, w_{k_{n}+2}^{I}, \ldots, w_{k-1}^{\prime}\right)
$$

It can be seen from formula (2) that the probability of a sentence is equal to the product of the probability of each word. There is an exponential relationship between the number of parameters and the value of $\mathrm{n}$. In theory, the larger the value of $\mathrm{n}$ is, the better. However, when the value of $\mathrm{n}$ is too large, the parameter space will be too large for the computer to calculate. When the value of $\mathrm{n}$ is too small, the long-distance language forms can't be effectively covered. The choice of $n$ value will affect the effect of the $\mathrm{N}$-gram model. Currently, Trigram $(\mathrm{M}=$ $3)$ and Bigram $(n=2)$ are used most. To ensure the efficiency of the algorithm, this paper chooses the Bigram model. That is to say, the occurrence probability of a word only depends on the front word.

$$
P(l)=P\left(w_{1}^{\prime}\right) \prod_{k=2}^{M} P\left(w_{k}^{\prime} \mid w_{k-1}^{\prime}\right)
$$

The iterative segmentation of compound words can reduce the ambiguity segmentation of long words. When the maximum matching segmentation method is used to segment a Chinese character string, the word frequency information in the training corpus can be used as the basis to judge whether the Chinese character string needs to be further segmented. Based on the automatic recognition of compound words, the iterative segmentation rule of compound words is added to determine whether a coarser-grained compound word needs to be further segmented into more fine-grained basic words. The specific iterative segmentation method is as follows.

For the Chinese character string $\mathrm{S}$ to be segmented, the segmentation result obtained in the process of the forward maximum matching method (FMM) and backward maximum matching method (BMM) is $\left(w_{1}, w_{2}, \ldots, w_{i}, \ldots\right)$. If the length of $w_{i}$ is greater than 3 , the frequency of $w_{i}$ in the training corpus is $N_{1}$. We continue to segment $w_{i}$ iteratively based on the standard dictionary. The result of segmentation is $w_{i}=\left(w_{i}^{1}, \ldots, w_{i}^{r_{i}}\right)$. The maximum frequency of ' $w_{i}^{1}, \ldots, w_{i}^{r_{i}}$, in the training corpus is $N_{2}$, and $N_{2}=\max \left(\operatorname{freq}\left(w_{i}^{1}\right), \ldots\right.$, freq $\left.\left(w_{i}^{r_{i}}\right)\right)$. freq $\left(w_{i}^{r_{i}}\right)$ is the frequency of $w_{i}^{r_{i}}$ in the training corpus, $1 \leq \mathrm{j} \leq r_{i}$. If $N_{2}$ is greater than $N_{1}$, the long entry $w_{i}$ is considered to be a combination. It needs to continue segmentation to get the final segmentation result $w_{i}^{1}, \ldots, w_{i}^{r_{i}}$. otherwise, $w_{i}$ remains unchanged. The above implementation process can be summarized as follows: 
$\left(w_{1}, w_{2}, \ldots, w_{i}\right)$ represents the word sequence obtained after initial segmentation, and $\left(w_{1}^{\prime}, w_{2}^{\prime}, \ldots, w_{k-1}^{\prime}\right)$ represents the word segmentation result after disambiguation.

For $w_{i}$, If $N_{1} \geq N_{2}$, then

$$
w_{k}^{\prime}=w_{i}
$$

If $N_{1}<N_{2}$, then

$$
w_{k}^{\prime}=w_{i}^{1}, w_{k+1}^{\prime}=w_{i}^{2}, \ldots, w_{k+r_{i}}^{\prime}=w_{i}^{r_{i}} .
$$

The string of Chinese characters to be segmented is marked as $\mathrm{S}$, and $l=\left(w_{1}^{\prime}, w_{2}^{\prime}, \ldots, w_{M}^{\prime}\right)$ is a possible segmentation result of $\mathrm{S}$. There are many schemes for $\mathrm{S}$ segmentation, and the result set is denoted by L (s). In this paper, when the bidirectional maximum matching method is used for Chinese word segmentation, two groups of word sequences $\left(l_{F}, l_{R}\right)$ are obtained by the forward and backward maximum matching algorithms. $\mathrm{L}(\mathrm{S})=\left\{l_{F}, l_{R}\right\}$. Based on the Markov hypothesis of the $\mathrm{N}$-gram language model, the optimal segmentation result $l^{*}$ of Chinese character string $\mathrm{S}$ is the optimal solution of probability equation (6).

$$
l^{*}=\arg \max _{i \in L(S)} P(l)=
$$

$\arg \max _{i \in L(S)} P\left(w_{1}^{\prime}\right) \prod_{k=2}^{M} P\left(w_{k}^{\prime} \mid w_{k_{0}+1}^{\prime}, w_{k_{0}+2}^{\prime}, \ldots, w_{k-1}^{\prime}\right)$

$2 \leq \mathrm{j} \leq \mathrm{M}, k_{0}=\max (\mathrm{k}-\mathrm{n}, 0)$. conditional probability:

$$
P\left(w_{k}^{\prime} \mid w_{k_{0}+1}^{\prime}, w_{k_{\mathrm{n}}+2}^{\prime}, \ldots, w_{k-1}^{\prime}\right)=\frac{P\left(w_{k_{0}+1^{\prime}}^{\prime}, w_{k_{0}+2^{, \ldots .},}^{\prime} w_{k-1}^{\prime}, w_{k}^{\prime}\right)}{P\left(w_{k_{n}+1}^{\prime}, w_{k_{n}+2^{\prime, \ldots,}}^{\prime} w_{k-1}^{\prime}\right)}
$$

maximum likelihood estimation:

$$
\widehat{P}\left(w_{k}^{\prime} \mid w_{k_{0}+1}^{\prime}, w_{k_{0}+2}^{I}, \ldots, w_{k-1}^{\prime}\right)=\frac{c\left(w_{k}^{\prime} \mid w_{k_{0}+1}^{\prime}, w_{k_{0}+2^{\prime}, \ldots,}^{\prime} w_{k-1}^{\prime}, w_{k}^{\prime}\right)}{\left(w_{k}^{\prime} \mid w_{k_{n}+1}^{\prime}, w_{\left.k_{n}+2^{\prime, \ldots,}, w_{k-1}^{\prime}\right)}^{\prime}\right)}(8)
$$

$C\left(w_{k}^{\prime} \mid w_{k_{0}+1}^{\prime}, w_{k_{\mathrm{n}}+2}^{\prime}, \ldots, w_{k-1}^{\prime}, w_{k}^{\prime}\right)$ is the frequency of the word sequence $w_{k_{0}+1}^{I}, w_{k_{0}+2}^{I}, \ldots, w_{k-1}^{I}, w_{k}^{I}$ in the training corpus.

For example, a famous scene in the romance of the Three Kingdoms is 'To Borrow Arrows with Thatched Boats'. So the Chinese text to be analyzed is '我需要一只船(I need a boat), 两岸有许多树(There are many trees on both sides), 灰蒙蒙 的天空 (Grey sky), 对面是敌人的阵地 (Opposite is the enemy's position)。'Using the bidirectional matching Chinese word segmentation method based on the $\mathrm{N}$-gram, we get '我 (I)/需要(need)/一只(a)/船(boat)/, 两岸(both sides)/有(have)/ 许多(many)/树(tree)/, 灰蒙蒙的(gray)/天空(sky)/, 对面 (opposite)/是(is)/敌人的(enemy)/阵地(position)/。
In order to verify the effectiveness of the Chinese word segmentation method based on $\mathrm{N}$-gram, the traditional $\mathrm{N}$-gram Chinese word segmentation method and the proposed method are respectively used for word segmentation on the test corpus. This paper proposes an evaluation standard $P_{n}$ to describe the accuracy of word segmentation results based on the total number of correct word segmentation.

$$
\mathrm{P}_{\mathrm{n}}=\frac{\sum_{i} \sum_{j} L\left(w_{i j}\right) I^{g}\left(w_{i j}\right)}{\mathrm{L}(\mathrm{V})}
$$

TABLE I. COMPARISON OF THE CORRECT NUMBER OF WORDS IN WORD SEGMENTATION RESULTS

\begin{tabular}{|l|c|c|c|}
\hline $\begin{array}{c}\text { Word segmentation } \\
\text { methods }\end{array}$ & $\begin{array}{c}\text { Parameter } \\
\text { setting }\end{array}$ & $\sum_{i} \sum_{j} \boldsymbol{L}\left(\boldsymbol{w}_{i j}\right) \boldsymbol{I}^{\boldsymbol{g}}\left(\boldsymbol{w}_{i j}\right)$ & $\mathbf{P}_{\mathbf{n}}$ \\
\hline \multirow{2}{*}{ N-gram model } & $\delta=1$ & 149491 & $81.13 \%$ \\
\cline { 2 - 4 } & $\delta=\mathbf{1 0}^{-\mathbf{5}}$ & 152868 & $82.96 \%$ \\
\hline \multirow{2}{*}{ BFEM } & $\delta=1$ & 157036 & $85.22 \%$ \\
\cline { 2 - 4 } & $\delta=\mathbf{1 0}^{-\mathbf{5}}$ & 161258 & $87.52 \%$ \\
\hline
\end{tabular}

The $\delta$ parameter represents the bonus. The total number of words in the test corpus is $L(V)=184263$. The column 3 of TABLE I represents the total number of correct entries according to the standard segmentation results. $P_{n}$ represents the ratio of the total number of words to be correctly segmented. As can be seen from TABLE I, when $\delta=1$, compared with the traditional $\mathrm{N}$-gram word segmentation method, the total number of words in the correct segmentation method presented in this paper increases by 7545 and the accuracy rate increases by $4.09 \%$. when $\delta=10^{-5}$, compared with the traditional $\mathrm{N}$-gram word segmentation method, the total number of words in the correct segmentation method presented in this paper increases by 8390 and the accuracy rate increases by $4.56 \%$.

\section{B. Extract the spatial relationship}

Chang A [39] proposes simple sentence extraction rules to analyze and extract the spatial relationship contained in the text. The expression of spatial semantic information is represented by three elements: Trajectory, Landmark, and Location Words. The Trajectory represents the entity objects. The Location Words represent the spatial position relationship. The Landmark represents the reference of the projectile. For example, ‘两岸有许多树(There are many trees on both sides.)'. In this statement, the '树(tree)' is the Trajectory. the '两岸 (both sides)' are the Landmark. the '边 (near-by)' is the Location Word. The spatial dependencies in the text are obtained by the Stanford parser.

- Extraction rules of simple sentences. A simple sentence contains a Trajectory, Landmark, and Location Words, and their extraction rules are shown in 'TABLE II'. For example, “两岸边有许多树(there are many trees on both sides.)'. The obtained dependency is shown in 'Fig. 1'.

TABLE II. SIMPLE SENTENCE EXTRACTION RULES 


\begin{tabular}{|l|c|}
\hline \multicolumn{1}{|c|}{ Extracting regular expressions } & result \\
\hline $\mathrm{nsubj}^{\wedge}($ root- $\downarrow \equiv$ nsubj- $\uparrow) \rightarrow \mathrm{nsubj}^{-} \downarrow$ & Trajectory \\
\hline $\mathrm{dobj}^{\wedge}($ root- $\downarrow \equiv$ dobj- $\uparrow) \rightarrow$ dobj- $\downarrow$ & Trajectory \\
\hline $\operatorname{lobj} \rightarrow$ lobj- $\downarrow$ & Landmark \\
\hline $\operatorname{lobj} \rightarrow \operatorname{lobj}-\uparrow$ & $\begin{array}{c}\text { Location } \\
\text { Words }\end{array}$ \\
\end{tabular}

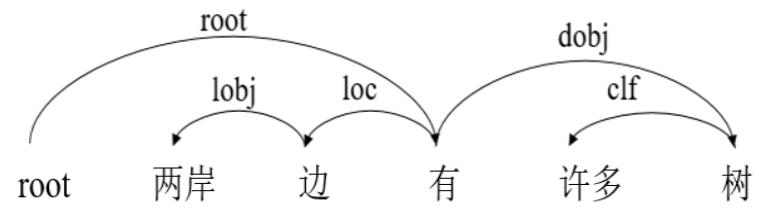

Fig. 1. The dependency of simple sentences.

The markings and meanings of dependency are shown in "TABLE III".

TABLE III. The MARKINGS AND MEANINGS OF DEPENDENCY

\begin{tabular}{|l|c|}
\hline \multicolumn{1}{|c|}{ markings } & meanings \\
\hline root & Core vocabulary, the root node \\
\hline lobj & Locating object \\
\hline dobj & Direct object \\
\hline loc & Positioning mark \\
\hline clf & Quantifier modification \\
\hline nsubj & Noun subject \\
\hline
\end{tabular}

- Trajectory recognition. The recognition of the Trajectory is related to whether there is an object in the sentence. If there is only a subject and no object in the sentence, the subject of the sentence is regarded as the Trajectory and the dependent word of the noun subject. If both the subject and the object are included in the sentence, the dependent word of the direct object is the Trajectory.

- Landmark recognition. Using the relationship of 'lobj', we can get the dependency word “两岸(both sides)" of the Landmark relationship 'lobj'.

- Location Words recognition. Reln (gov, dep) stands for binary dependency. 'Reln' stands for relationship name. 'gov' stands for the central word. 'dep' stands for dependency. The center word of the locating object (有, 树) in the binary relationship is “有”, which can be found through the locating object.

\section{3D SCENE CONSTRUCTION METHOD}

This paper proposes a 3D scene construction method based on a spatial relation case-base, as shown in 'Fig. 2'. Firstly, the semantic scene description of the user input text scene is analyzed. Semantic analysis provides support for retrieval based on semantic. So, we need to label the scene elements in the scene. Annotation is the basis of model retrieval based on semantic analysis. Then, according to the description of semantic features, Scene objects and spatial relationship information are extracted. The corresponding model is retrieved from the case-base, which provides support for retrieval based spatial relation. The spatial relations extracted from semantics are stored in the spatial relation template library in the form of triples.

This method that is based on the object spatial geometric constraints of the virtual physical world automatically guides the objects to be placed in the spatial, which is convenient for users to layout the complex scene quickly, conveniently, and reasonably. A reasonable and effective scene design must satisfy the relevant constraints. spatial relationship is a kind of spatial constraint between spatial objects, which is mainly used for scene layout. Different spatial relationships express the relationship between spatial objects in different angles and different measurement methods.

A scene is described by two-tuple ( $\mathrm{Scene}=\left\langle\mathrm{SE}, R_{S E}\right\rangle$ ). SE is a collection of scene objects, and $R_{S E}$ is the relationship on SE. Scene objects are the entities that make up the scenes, such as banks, trees, rivers, boats, etc. The relationship of scene objects is the spatial relationship between objects. In this paper, the spatial relation is described by a triple $(\mathrm{O}, \mathrm{X}, \mathrm{Y})$ : $\mathrm{X}$ and $\mathrm{Y}$ are scene objects. $\mathrm{O}$ is the spatial semantic constraint between $\mathrm{X}$ and $\mathrm{Y} . \mathrm{X}$ is the Trajectory in spatial relationship. $\mathrm{Y}$ is the Landmark of spatial relations. For example, (上,树,两岸)(up, tree, both sides) expresses the spatial constraint of '两岸上的 树 (Trees on both banks)', and (上, 船, 河)(up, boat, river) expresses the spatial constraint of '河上有只船 (There is a boat on the river)'.

\section{A. Case-base and spatial relationship template library are established in $3 D$ scene}

The initial scene example often contains a lot of disordered and unnecessary information, which needs to be filtered and reorganized. Before spatial relations are retrieved, the reprocessed models can avoid system jam and insufficient memory caused by loading a large number of models during retrieval. The scene attributes semantic description, and the important scene elements in the scene also need to be annotated according to the annotation method of the scene objects. Scene elements are part of the scene. All operations on the scene will eventually be converted to operations on the scene elements. Therefore, to operate the scene elements more conveniently and effectively, it is necessary to label the scene elements.

In addition to labeling the geometric information and appearance information of scene elements, we also need to consider the semantic information contained in scene elements. To describe the spatial relationship between objects accurately and conveniently, we must label each object in the scene correctly. The types of tags include spatial tags and object tags, which are presented in the form of text annotation. Annotated objects are stored in different case-bases according to different scenes to provide the required models for automatic modeling. Frequently used scene objects are stored in the template library in the form of triples (objects and spatial annotation texts), so that the spatial constraints are regarded as the minimum module of the scene. This method not only accelerates the speed of scene construction, but also improves the rationality of scene layout. 


\section{B. Semantic analysis and searching case-base}

The scene semantic description is analyzed, and according to the results, different scenes match the corresponding casebase (semantic description texts match object annotation in the case-base). The spatial relationship contained in the texts are extracted and searched in the spatial relationship template library in the form of triples.

\section{Matching spatial relationship template library}

The spatial constraints searched in the spatial relationship template library. According to the spatial constraints, the model objects imported from the case-base can be placed in the scene under the spatial constraints.

\section{XML file storage 3D scene model information and related attributes.}

In this paper, the models are reprocessed by text annotation to facilitate the retrieval of model objects later. The annotation of the model includes the model information and its related attributes and will be stored in an XML file.

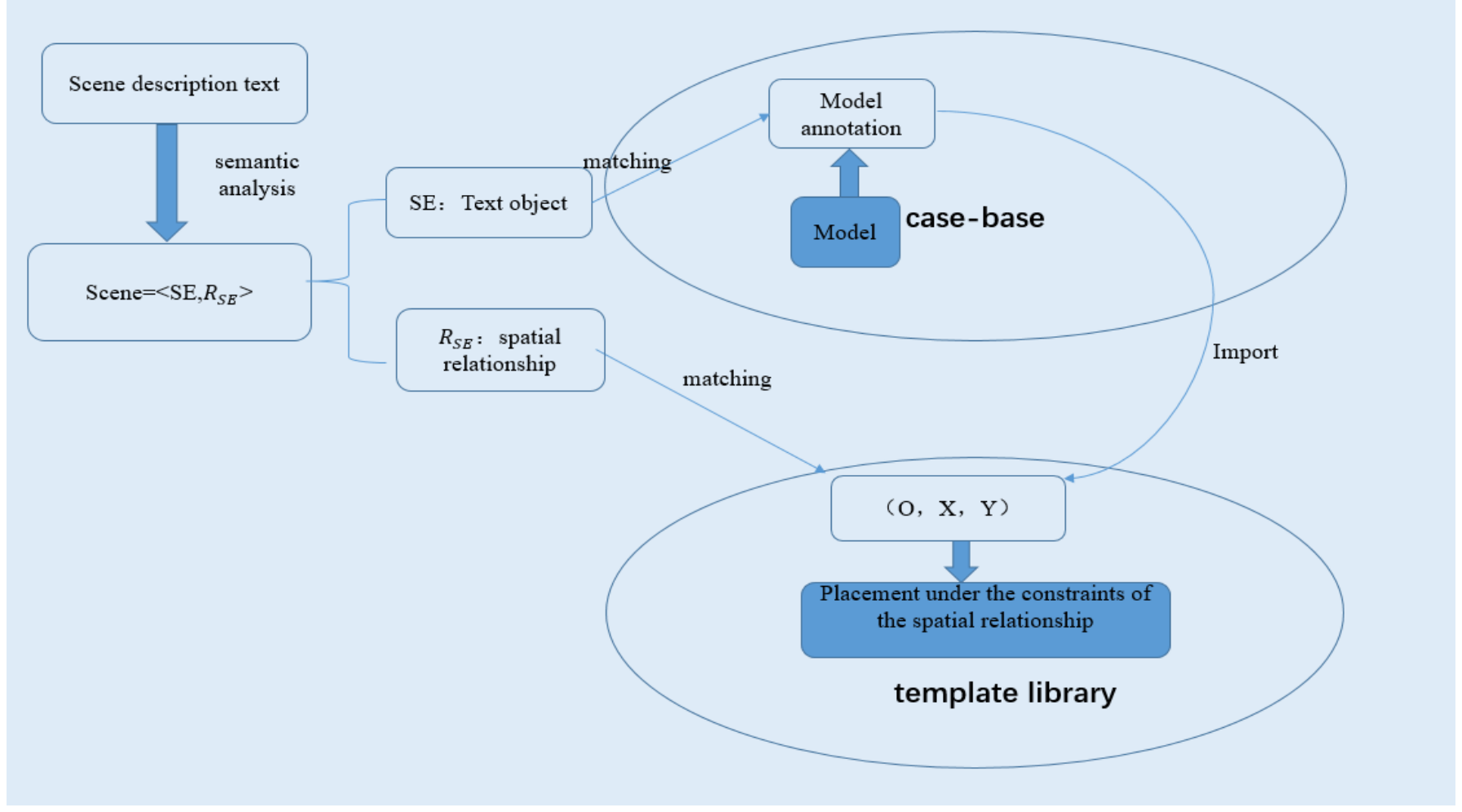

Fig. 2. 3D scene construction based on spatial relation case-base.

\section{APPLICATION IN STRATEGY GAME}

Strategy game provides players with an environment in which they can use their brains to think about problems and deal with more complex things. It allows players to freely control, manage and use the characters or things in the game. Through this free means and the ways that players use their brains to come up with against the enemy, they can achieve the goal required in the game. Strategy game provides players with an environment in which they can use their brains to pondering problems and deal with more complex things.

There are many classic plots, Intrigue and wisdom, and heroic individuals in the masterpiece romance of the Three Kingdoms. Its vigorous and unrestrained temperament between the words, which makes the famous work become a welldeserved treasure in the history of Chinese literature. Take the story of 'To Borrow Arrows with Thatched Boats' as the background of the game. Players build 3D scenes by semanticdriven 3D scene construction based on spatial relation and case-base in the strategy game and pay attention to how to use strategy to build the scene to achieve victory.
For example, the text 1: ‘我需要一只船, 两岸有许多 树, 灰蒙蒙的天空, 对面是敌人的阵地。'(I need a boat, there are many trees on both sides, the gray sky and opposite is the enemy's position.) Its 3D scene is shown in 'Fig. 3'. 


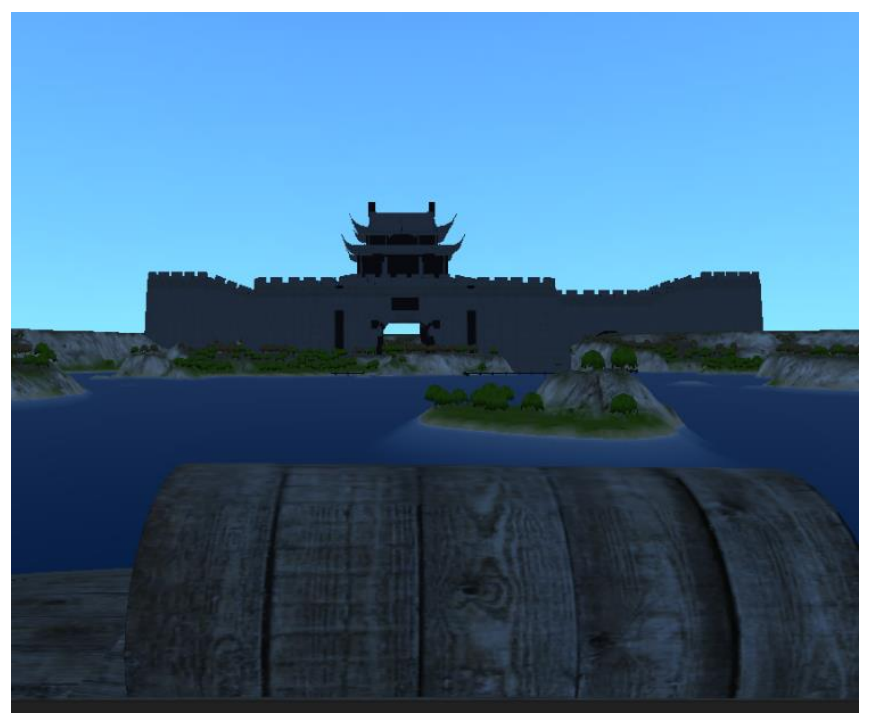

Fig. 3. The 3D scene constructed by text 'under the gray sky'.

the text 2: ‘我需要一只船, 两岸有许多树, 万里无云的 天空, 对面是敌人的阵地。'(I need a boat, there are many trees on both sides, the cloudless sky and opposite is the enemy's position.) Its 3D scene is shown in 'Fig. 4'.

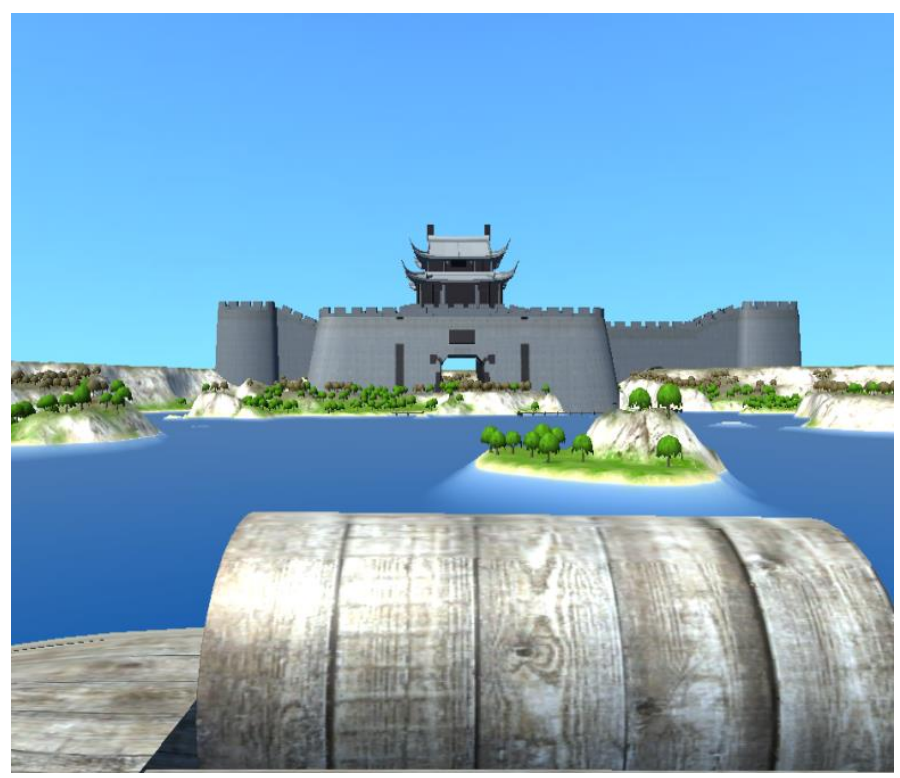

Fig. 4. The 3D scene constructed by text 'under the cloudless sky'.

the text 3: “万里无云的天空下, 敌人的阵地周围地势崎 岖, 地上是草和灌木从丛。'(Under the cloudless sky, the terrain around the enemy's position was rugged, with grass and bushes on the ground.) Its 3D scene is shown in 'Fig. 5'.

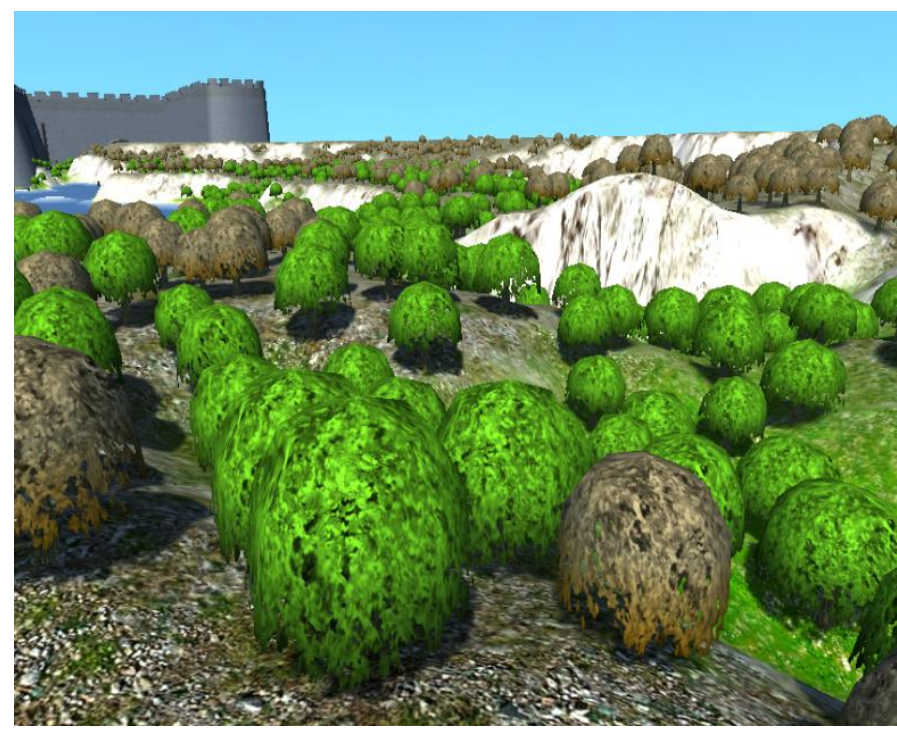

Fig. 5. The 3D scene constructed by text 'with grass and bushes'.

\section{CONCLUSION}

In this paper, we propose a new method of semanticdriven 3D scene construction based on spatial relation and case-base. The processing of natural language includes bidirectional matching Chinese word segmentation method based on $\mathrm{N}$-gram model. This segmentation result is very helpful for the ambiguous words segmentation. The extraction rules of simple sentences are used to extract the spatial relations contained in the texts. The method of 3D scene construction based on spatial relation and case-base realizes the automatic construction of the scene. Strategy games are beneficial to the development of the brain. Taking the Three Kingdoms as the background of the game can not only increase the interest of the game, but also help people understand history. We apply it to a strategy game, which makes the players pay more attention to the strategy itself.

The method of 3D scene construction based on spatial relation and case-base improves the automation, the usability and quickness of 3D scene production. In addition, this method reduces the production cost of $3 \mathrm{D}$ scene and repeated labor, shortens the period of production.

\section{REFERENCES}

[1] S. Meiling, F. Zhengyong, "Digital Technology and Application," Virtual Reality Technology. 2015.

[2] E. Bastug, M. Bennis, M. Medard, et al. "Toward Interconnected Virtual Reality: Opportunities," Challenges, and Enablers. IEEE Communications Magazine, 2017,55(6): pp.110-117.

[3] D. Julia, G. W. Alpers, H. M. Peperkorn, et al. The impact of perception and presence on emotional reactions: a review of research in virtual reality. Frontiers in Psychology, 2015, 6: 26.

[4] Y. J. Lan, "Immersion, interaction, and experience-oriented learning: Bringing virtual reality into FL learning," Language Learning \& Technology, 2020, 24.

[5] D. Schofield, B. Denby, D. Mcclarnon, "Computer graphics and virtual reality in the mining industry," International Journal of Rock Mechanics \& Mining Science \& Geomechanics Abstracts, 1994, 32(4): 284-286.

[6] N. Ayache, "Medical Computer Vision, Virtual Reality and Robotics: Promising Research Tracks," Image \& Vision Computing, 2011, 13(4): 295-313. 
[7] A. Chang, M. Savva, C D. Manning, "Interactive Learning of Spatial Knowledge for Text to 3D Scene Generation," Proceedings of the Workshop on Interactive Language Learning, Visualization, and Interfaces, 2014.

[8] M. Fisher, P. Hanrahan, "Context-Based Search for 3D Models," Acm Transactions on Graphics, 2010, 29(6): pp.1-10.

[9] K A. Masood, A K. Rahat. An approach to text analytics and text mining in multilingual natural language processing. 2021.

[10] D. Caitli, A. Theresa, Koleck, E. Philip, Bourne, et al. "A systematic review of natural language processing and text mining of symptoms from electronic patient-authored text data," 2019, 125: pp.37-46.

[11] K. Stephen, "Strategy Games To Enhance Problem-solving Ability In Mathematics," Alfred S Posamentier. 2016.

[12] N. Nikitakos, I. Sirris, D. Dalaklis, et al. "Game-based learning for maritime education and training: the case of Trader of the World," 2017, 16(2): pp.265-291.

[13] M. Fisher, D. Ritchie, M. Savva, et al. "Example-based synthesis of 3D object arrangements," Acm Transactions on Graphics (S0730-0301), 2012, 31(6): pp.1-11.

[14] D. Gallup, J. M. Frahm, M. Pollefeys, "A heightmap model for efficient 3d reconstruction from street-level video," Proc. Int. Conf. on 3D Data Processing, Visualization and Transmission, 2010. Paris, May, 2010: pp.1-10.

[15] D. Picca, D. Jaccard, G. Eberlé, "Natural Language Processing in Serious Games: A state of the art," International Journal of Serious Games, 2015, 2(3)

[16] T. C. Guetterman, T. Chang, M. Dejonckheere, et al. "Augmenting Qualitative Text Analysis with Natural Language," Journal of Medical Internet Research, 2018, 20(6).

[17] C. Gong, Z. Li, M. Zhang, et al. "Multi-Grained Chinese Word Segmentation," Proceedings of the 2017 Conference on Empirical Methods in Natural Language Processing, 2017.

[18] R. Řehưřek, P. Sojka, "Software Framework for Topic Modelling with Large Corpora," Proceedings of the LREC 2010 Workshop on New Challenges for NLP Frameworks, 2010.

[19] J. Daniel, "Speech and Language Processing: An Introduction to Natural Language Processing, Computational Linguistics, and Speech Recognition," the Posts and Telecommunications Press, 2010.

[20] C.C. Yang, "Search Engines Information Retrieval in Practice," Journal of the American Society for Information ence \& Technology, 2014, 61(2): pp.430-430.

[21] A. Spink, T. Saracevic, "Interaction in information retrieval: Selection and effectiveness of search terms," Journal of the Association for Information Science \& Technology, 2010, 48(8): pp.741-761.

[22] J. Atkinson-Abutridy, C. Mellish, S. Aitken, "Combining Information Extraction with Genetic Algorithms for Text Mining," IEEE Intelligent Systems, 2017, 19(3): pp.22-30.

[23] K. F. Wong, W. Li, R. Xu, et al. "Introduction to Chinese Natural Language Processing," Synthesis Lectures on Human Language Technologies, 2010, 2(1)

[24] D. Picca, D. Jaccard, G. Eberlé, "Natural Language Processing in Serious Games: A state of the art" International Journal of Serious Games, 2015, 2(3)
[25] T. C. Guetterman, T. Chang, M. Dejonckheere, et al. "Augmenting Qualitative Text Analysis with Natural Language Processing: Methodological Study," Journal of Medical Internet Research, 2018, 20(6).

[26] C. Gong, Z. Li, M. Zhang, et al. "Multi-Grained Chinese Word Segmentation" Proceedings of the 2017 Conference on Empirical Methods in Natural Language Processing, 2017.

[27] Y. Su, S. Yang, H. Sun, et al. "Exploiting Relevance Feedback in Knowledge Graph Search," The 21th ACM SIGKDD International Conference on Knowledge Discovery and Data Mining. ACM, 2015.

[28] K. Natthawut, I. Ryutaro, "An Automatic Knowledge Graph Creation Framework from Natural Language Text," Ieice Transactions on Information \& Systems, 2018, 101(1): pp.90-98.

[29] J. Rui, S. Yu, H. Yan, et al. "Visualization and Forecast Analysis of Science and Technology Intelligence Based on Knowledge Graph," 2018 17th International Symposium on Distributed Computing and Applications for Business Engineering and Science (DCABES). IEEE Computer Society, 2018.

[30] W. Shen, X. Li, X. Li, "Processing and Representation of Ambiguous Words in Chinese Reading: Evidence from Eye Movements," Frontiers in Psychology, 2016, 7: 1713.

[31] N. Vassilas, G. Miaoulis, D. Chronopoulos, et al. "MultiCAD -GA: A System for the Design of 3D Forms Based on Genetic Algorithms and Human Evaluation," Hellenic Conference on Ai: Methods and Applications of Artificial Intelligence. Springer-Verlag, 2002: pp.203214.

[32] K. Ghassan, G. Véronique, R. Caubet, "DEM2ONS : A High Level Declarative Modeler For 3D Graphics Applications," Proceedings of the International Conference on Imaging Science Systems and Technology. CISST, 97, Las Vegas, 1997: pp.149-154.

[33] K. Xu, J. Stewart, E. Fiume, "Constraint-based Automatic Placement for Scene Composition" Proceedings - Graphics Interface, 2002: pp.25-34.

[34] T. Tim, B. Rafael, et al. "The role of semantics in games and simulations," Computers in Entertainment, 2009.

[35] Y. Akazawa, Y. Okada, K. Niijima, "Automatic 3D scene generation based on contact constraints," 2016.

[36] S. Sanchez, O. Le Roux, Luga H, et al. "Constraint-based 3D object layout using a genetic algorithm," 2016.

[37] M. Larive, O. Le Roux, V. Gaildrat. "Using meta-heuristics for constraint-based 3D object layout" 2016

[38] A H. Siagian, M. Aritsugi, "Robustness of Word and Character N-gram Combinations in Detecting Deceptive and Truthful Opinions" Journal of Data and Information Quality (JDIQ), 2020.

[39] A. Chang, W. Monroe, M. Savva, et al. "Text to 3D Scene Generation with Rich Lexical Grounding," Computer Science, 2015.

[40] A. Dey, M. Jenamani, J. J. Thakkar, "Senti-N-Gram: An n-gram lexicon for sentiment analysis," Expert Systems with Applications, 2018.

[41] Y. Chang, "Interactive Storytelling of 'Romance of the Three Kingdoms' Series in Simulation Game,” 2008, 15: pp.37-54.

[42] W. Shiang-Kwei, "Effects of Playing a History-Simulation Game: Romance of Three Kingdoms," 2010, 2(2).

[43] Y. GUO, H. WANG, J. VAN GENABITH. Dependency-based n-gram models for general purpose sentence realization. Natural Language Engineering, 2011, 17(4), 455-483. 